Pacific

Journal of

Mathematics

INDEX THEORY AND TOEPLITZ ALGEBRAS ON ONE-PARAMETER SUBGROUPS OF LIE GROUPS

EFTON PARK

Volume $158 \quad$ No. 1

March 1993 


\title{
INDEX THEORY AND TOEPLITZ ALGEBRAS ON ONE-PARAMETER SUBGROUPS OF LIE GROUPS
}

\author{
EFTON PARK
}

\begin{abstract}
We form the Toeplitz $C^{*}$-algebra $\mathscr{T}(G ; X)$ associated to the one-parameter subgroup $\exp (t X)$ defined by a left-invariant vector field $X$ on a compact Lie group $G$. We compute the $K$-theory of $\mathscr{T}(G ; X)$ and its commutator ideal $\mathscr{C}(G ; X)$. We also define an abstract analytical index for $\mathscr{T}(G ; X)$ and show that this analytical index can be computed in terms of topological data.
\end{abstract}

Introduction. Let $G$ be a compact Lie group, let $X$ be a non-zero left-invariant vector field on $G$, and let $\frac{1}{i} L_{X}$ denote the Lie derivative with respect to $X$. The operator $\frac{1}{i} L_{X}$ extends to an unbounded self-adjoint operator on $L^{2}(G)$; let $P$ denote the positive spectral projection of $\frac{1}{i} L_{X}$. Next, each continuous complex-valued function $\phi$ on $G$ gives rise to a bounded operator $M_{\phi}$ on $L^{2}(G)$ via multiplication. We form a $C^{*}$-algebra $\mathscr{T}(G ; X)$ that is generated by the set $\left\{P M_{\phi}: \phi \in C(G)\right\}$, and we call this algebra the Toeplitz algebra of $X$. In this paper we study $\mathscr{T}(G ; X)$, and in particular, we look at how $\mathscr{T}(G ; X)$ depends on the geometry and topology of $G$ and the choice of $X$.

Our interest in $\mathscr{T}(G ; X)$ stems from two sources. First, several authors have recently obtained very nice results relating Toeplitz algebras on flows to the topology of the flows $[2,8]$. In our case, the vector field $X$ determines a flow on $G$, and $\mathscr{T}(G ; X)$ is the Toeplitz algebra of this flow.

Another important reason for studying $\mathscr{T}(G ; X)$ is that this algebra contains information about the self-adjoint operator $\frac{1}{i} L_{X}$. In [4], the authors used Toeplitz algebra techniques to study self-adjoint operators that are elliptic along the leaves of a foliation. In our case, the cosets of $\exp (t X)$ foliate $G$, and $\frac{1}{i} L_{X}$ is elliptic along the leaves of this foliation.

In all of the work mentioned above, the authors required their flows and foliations to be minimal. Our foliations are typically not minimal, and in fact can be very far from being minimal. Thus, we must develop techniques for studying $\mathscr{T}(G ; X)$ that do not require minimality. 
To see how to proceed, let us consider a simple example. Let $H=$ $\exp (t X)$ be the one-parameter subgroup generated by $X$, and let $\bar{H}$ be its closure. Suppose that $G$ is topologically the product of $\bar{H}$ and a compact space $B$. Then $\mathscr{T}(G ; X)$ is isomorphic to $C(B) \otimes$ $\mathscr{T}(\bar{H} ; X)$. Moreover, since $H$ is a connected abelian Lie group, so is $\bar{H}$, and therefore $\bar{H}$ is isomorphic to some torus $\mathbb{T}^{n}$. The structure of the algebra $\mathscr{T}\left(\mathbb{T}^{n} ; X\right)$ was worked out in [5] and [6], and hence we know the structure of $\mathscr{T}(G ; X)$ when $G$ is a product. Of course, $G$ cannot always be written as a product. However, $G$ is locally a product. More precisely, let $B$ be the homogeneous space $G / \bar{H}$. Then $G$ is a fiber bundle over $B$ with fiber $\bar{H}$. It is this local product structure that we use to analyze $\mathscr{T}(G ; X)$. We will show that $\mathscr{T}(G ; X)$ is isomorphic to the $C^{*}$-algebra of sections of a continuous field of Toeplitz algebras for which the subgroup is dense. Using this approach, we can say a great deal about $\mathscr{T}(G ; X)$ in the general case.

This paper is organized as follows. In $\S 1$, we define the Toeplitz algebra $\mathscr{T}(G ; X)$ and show that it is isomorphic to the $C^{*}$-algebra of sections of a continuous field of Toeplitz algebras. In $\S 2$, we compute the $K$-theory of $\mathscr{T}(G ; X)$. In $\S 3$, we prove that the quotient of $\mathscr{T}(G ; X)$ by its commutator ideal $\mathscr{C}(G ; X)$ is isomorphic to $C(G)$, and we apply $K$-theory to the corresponding exact sequence to obtain a homomorphism from the $K$-theory of $C(G)$ to that of $\mathscr{C}(G ; X)$; we call this homomorphism the analytical index. In $\S 4$ we consider the Lie group $G$ as a fiber bundle over $G / \overline{\exp (t X)}$, and we define a topological index. In $\S 5$ we compute the $K$-theory of $\mathscr{C}(G ; X)$ and show that the analytical index and the topological index can be identified. Finally, in $\S 6$ we consider a "smoothed" version $\mathscr{T}_{\varepsilon}(G ; X)$ of the Toeplitz algebra and state some results for $\mathscr{T}_{\varepsilon}(G ; X)$.

1. The Toeplitz algebra $\mathscr{T}(G ; X)$. Let $G$ be a compact Lie group, let $X$ be a nonzero left-invariant vector field on $G$, and let $L_{X}$ denote the Lie derivative with respect to $X$. It is well-known that $\frac{1}{i} L_{X}$ defines an unbounded self-adjoint differential operator on $L^{2}(G)$, where $G$ is endowed with (left) Haar measure. Let $P$ be the positive spectral projection of $\frac{1}{i} L_{X}$; that is, use the functional calculus for self-adjoint operators to define $P=\chi_{[0, \infty)}\left(\frac{1}{i} L_{X}\right)$, where $\chi_{[0, \infty)}$ denotes the characteristic function of the positive real numbers. Now, a continuous complex-valued function $\phi$ on $G$ defines a bounded operator $M_{\phi}$ on $L^{2}(G)$ via multiplication: $M_{\phi}(f)=\phi f$. With this in mind, we make the following definition: 
Definition. The Toeplitz algebra of $X$, denoted $\mathscr{T}(G ; X)$, is the $C^{*}$-subalgebra of $\mathscr{L}\left(L^{2}(G)\right)$ generated by $\left\{P M_{\phi}: \phi \in C(G)\right\}$.

We now wish to show that $\mathscr{T}(G ; X)$ is isomorphic to the $C^{*}$ algebra of sections of a certain continuous field of $C^{*}$-algebras. We refer the reader to [3] for the definition and properties of continuous fields of $C^{*}$-algebras.

Associated to the vector field $X$ is the one-parameter subgroup $H=$ $\exp (t X)$ of $G$. Its closure $\bar{H}$ is also a subgroup; let $B$ be the quotient space $G / \bar{H}$, and let $\pi$ denote the natural quotient map from $G$ to $B$. With this map, $G$ is a fiber bundle over $B$ with fiber $\bar{H}$.

The group $\bar{H}$ is itself a Lie group, so we can endow $\bar{H}$ with its own Haar measure. Moreover, we can translate this measure to put a measure on each coset $\pi^{-1}(b)$ of $\bar{H}$. The Lie derivative with respect to $X$ acts on each Hilbert space $L^{2}\left(\pi^{-1}(b)\right)$, and we will denote this operator by $\frac{1}{i} L_{X}^{b}$. Also, define $P^{b}=\chi_{[0, \infty)}\left(\frac{1}{i} L_{X}^{b}\right)$ on $L^{2}\left(\pi^{-1}(b)\right)$ for each $b$ in $B$. Next, for each $b \in B$, let $\mathscr{T}\left(\pi^{-1}(b) ; X\right)$ denote the $C^{*}$-subalgebra of $\mathscr{L}\left(L^{2}\left(\pi^{-1}(b)\right)\right)$ generated by $\left\{P^{b} M_{\psi}: \psi \in\right.$ $\left.C\left(\pi^{-1}(b)\right)\right\}$.

Define a continuous field of $C^{*}$-algebras over $B$ in the following manner. Form the direct product $\prod_{b \in B} \mathscr{T}\left(\pi^{-1}(b) ; X\right)$, and for each $\phi \in C(G)$, let $\gamma_{\phi} \in \prod_{b \in B} \mathscr{T}\left(\pi^{-1}(b) ; X\right)$ be defined by $\gamma_{\phi}(b)=$ $P^{b} M_{\phi^{b}}$, where $\phi^{b}$ is the restriction of $\phi \in C(G)$ to $\pi^{-1}(b)$. Let $\Gamma$ be the algebra of continuous sections of $\prod_{b \in B} \mathscr{T}\left(\pi^{-1}(b) ; X\right)$ generated by $\left\{\gamma_{\phi}: \phi \in C(G)\right\}$.

Proposition 1.1. $\left(\prod_{b \in B} \mathscr{T}\left(\pi^{-1}(b) ; X\right), \Gamma\right)$ is a continuous field of $C^{*}$-algebras over $B$.

Proof. The only nonobvious point to check is that for each $\gamma$ in $\Gamma$, the function $b \longmapsto\|\gamma(b)\|$ is continuous. Since every $\gamma \in \Gamma$ can be uniformly approximated by finite products and sums of the sections $\gamma_{\phi}$, it suffices to show that for each $\phi \in C(G)$, the function $b \longmapsto$ $\left\|\gamma_{\phi}(b)\right\|$ is continuous. But $\left\|\gamma_{\phi}(b)\right\|=\left\|\phi^{b}\right\|$ [7], from which continuity follows immediately.

Proposition 1.2. The Toeplitz algebra $\mathscr{T}(G ; X)$ is isomorphic to the $C^{*}$-algebra of sections of the continuous field

$$
\left(\prod_{b \in B} \mathscr{T}\left(\pi^{-1}(b) ; X\right), \Gamma\right) .
$$


Proof. Push forward Haar measure on $G$ via $\pi$ to obtain a measure $\mu$ on $B$. For each continuous function $\phi$ in $L^{2}(G)$, there corresponds an element $\left(\phi^{b}\right)_{b \in B}$ of $\prod_{b \in B} L^{2}\left(\pi^{-1}(b)\right)$, and this correspondence extends to an isometric isomorphism from $L^{2}(G)$ to a direct integral $\int L^{2}\left(\pi^{-1}(b)\right) d \mu$ of Hilbert spaces. Let $U$ be a unitary implementing this isomorphism. Then $U^{*} P U=\left(P^{b}\right)_{b \in B}$ and $U^{*} M_{\phi} U=\left(M_{\phi^{b}}\right)_{b \in B}$ for every $\phi$ in $C(G)$, so $U$ implements an isomorphism from $\mathscr{T}(G ; X)$ to the $C^{*}$-algebra of sections of $\left(\prod_{b \in B} \mathscr{T}\left(\pi^{-1}(b) ; X\right), \Gamma\right)$.

Definition. Let $Y$ be a closed subset of $B$, and let

$$
\left(\prod_{b \in Y} \mathscr{T}\left(\pi^{-1}(b) ; X\right), \Gamma_{\mid Y}\right)
$$

be the continuous field over $Y$ that is the restriction of

$$
\left(\prod_{b \in B} \mathscr{T}\left(\pi^{-1}(b) ; X\right), \Gamma\right)
$$

to $Y$. Then $\mathscr{T}\left(\pi^{-1}(Y) ; X\right)$ is the $C^{*}$-algebra of sections of

$$
\left(\prod_{b \in Y} \mathscr{T}\left(\pi^{-1}(b) ; X\right), \Gamma_{\mid Y}\right) \text {. }
$$

With the definition above, the following two corollaries are immediate.

CoROllaRY 1.3. The correspondence $A \longmapsto \mathscr{T}\left(\pi^{-1}(A) ; X\right)$ is a contravariant functor from the category of closed subsets of $B$ and inclusion maps to the category of Toeplitz algebras over closed subsets of $B$ and homomorphisms.

Corollary 1.4. Let $A_{1}$ and $A_{2}$ be closed subsets of $B$. Then $\mathscr{T}\left(\pi^{-1}\left(A_{1} \cup A_{2}\right) ; X\right)$ is isomorphic to the $C^{*}$-algebra

$$
\left\{\left(T_{1}, T_{2}\right) \in \mathscr{T}\left(\pi^{-1}\left(A_{1}\right) ; X\right) \oplus \mathscr{T}\left(\pi^{-1}\left(A_{2}\right) ; X\right): r_{1}\left(T_{1}\right)=r_{2}\left(T_{2}\right)\right\},
$$

where $r_{1}$ and $r_{2}$ are the restriction homomorphisms from $\mathscr{T}\left(\pi^{-1}\left(A_{1}\right) ; X\right)$ and $\mathscr{T}\left(\pi^{-1}\left(A_{2}\right) ; X\right)$ to $\mathscr{T}\left(\pi^{-1}\left(A_{1} \cap A_{2}\right) ; X\right)$.

2. The $K$-theory of $\mathscr{T}(G ; X)$. In this section, we compute the $K$ theory of $\mathscr{T}(G ; X)$. We begin by considering the Toeplitz algebra in the special case where the one-parameter group $H=\exp (t X)$ is dense in $G$, so $G=\bar{H}$ and $\mathscr{T}(G ; X)=\mathscr{T}(\bar{H} ; X)$. In this case $G$ is the 
closure of a connected abelian group and is therefore an $n$-torus $\mathbb{T}^{n}$ for some $n$. This situation was studied in [5] and [6], and it is shown there that $K_{0}(\mathscr{T}(G ; X)) \cong \mathbb{Z}$ and $K_{1}(\mathscr{T}(G ; X)) \cong 0$.

We now consider the general case. Let $\pi^{*}$ be the natural homomorphism from $C(B)$ to $C(G)$. Then for a closed subset $A$ of $B$, define $\alpha: C(A) \longrightarrow \mathscr{T}\left(\pi^{-1}(A) ; X\right)$ by $\alpha(\phi)=P M_{\pi^{*}(\phi)}$. Note that since $\pi^{*}(\phi)$ is constant when restricted to sets of the form $\pi^{-1}(b)$, $\frac{1}{i} L_{X}$, and hence $P$, commutes with $M_{\pi^{*}(\phi)}$ for all $\phi \in C(A)$. Thus $\alpha$ is a homomorphism.

Theorem 2.1. The map $\alpha: C(B) \longrightarrow \mathscr{T}(G ; X)$ induces an isomorphism $\alpha_{*}: K_{*}(C(B)) \longrightarrow K_{*}(\mathscr{T}(G ; X))$, whence $K_{*}(\mathscr{T}(G ; X)) \cong$ $K^{*}(B)$.

Proof. Let $H=\exp (t X)$, and let $A$ be a closed subset over which $G$ is trivial. Then $\mathscr{T}\left(\pi^{-1}(A) ; X\right) \cong C(A) \otimes \mathscr{T}(\bar{H} ; X)$, and under this isomorphism, $\alpha(\phi)=\phi \otimes I$. The natural inclusion of $\mathbb{C}$ into $\mathscr{T}(\bar{H} ; X)$ induces an isomorphism in $K$-theory $[5,6]$, so $\alpha$ also induces an isomorphism in $K$-theory.

Suppose that $A_{1}$ and $A_{2}$ are closed subsets of $B$ such that $\alpha$ induces isomorphisms $K_{*}\left(C\left(A_{1}\right)\right) \cong K_{*}\left(\mathscr{T}\left(\pi^{-1}\left(A_{1}\right)\right) ; X\right)$ and $K_{*}\left(C\left(A_{2}\right)\right)$ $\cong K_{*}\left(\mathscr{T}\left(\pi^{-1}\left(A_{2}\right) ; X\right)\right)$. Then we also have an isomorphism from $K_{*}\left(C\left(A_{1} \cap A_{2}\right)\right)$ to $K_{*}\left(\mathscr{T}\left(\pi^{-1}\left(A_{1} \cap A_{2}\right) ; X\right)\right)$. By Corollary 1.4, we can apply the Mayer-Vietoris sequence in $K$-theory [10], and for $i=0,1$, we have the following commutative diagram with exact columns:

$$
\begin{array}{ccc}
K_{1-i}\left(C\left(A_{1}\right) \oplus C\left(A_{2}\right)\right) & \longrightarrow & K_{1-i}\left(\mathscr{T}\left(\pi^{-1}\left(A_{1}\right) ; X\right) \oplus \mathscr{T}\left(\pi^{-1}\left(A_{2}\right) ; X\right)\right) \\
\downarrow & & \\
K_{1-i}\left(C\left(A_{1} \cap A_{2}\right)\right) & \longrightarrow & K_{1-i}\left(\mathscr{T}\left(\pi^{-1}\left(A_{1} \cap A_{2}\right) ; X\right)\right) \\
\downarrow & & \downarrow \\
K_{i}\left(C\left(A_{1} \cup A_{2}\right)\right) & \longrightarrow & K_{i}\left(\mathscr{T}\left(\pi^{-1}\left(A_{1} \cup A_{2}\right) ; X\right)\right) \\
\perp & & \downarrow \\
K_{i}\left(C\left(A_{1}\right) \oplus C\left(A_{2}\right)\right) & \longrightarrow & K_{i}\left(\mathscr{T}\left(\pi^{-1}\left(A_{1}\right) ; X\right) \oplus \mathscr{T}\left(\pi^{-1}\left(A_{2}\right) ; X\right)\right) \\
\perp & & \\
K_{i}\left(C\left(A_{1} \cap A_{2}\right)\right) & \longrightarrow & K_{i}\left(\mathscr{T}\left(\pi^{-1}\left(A_{1} \cap A_{2}\right) ; X\right)\right) .
\end{array}
$$


An application of the five lemma shows that

$$
\alpha_{*}: K_{*}\left(C\left(A_{1} \cup A_{2}\right)\right) \longrightarrow K_{*}\left(\mathscr{T}\left(\pi^{-1}\left(A_{1} \cup A_{2}\right) ; X\right)\right)
$$

is an isomorphism, and since $B$ can be written as the union of a finite number of closed sets over which $G$ is trivial, an induction argument shows that

$$
\alpha_{*}: K_{*}(C(B)) \longrightarrow K_{*}(\mathscr{T}(G ; X))
$$

is an isomorphism. Finally, composition with the natural isomorphism $K_{*}(C(B)) \cong K^{*}(B)$ gives the desired result.

3. The analytical index. In this section, we construct a short exact sequence that involves the Toeplitz algebra, and we use this short exact sequence to define an abstract analytical index for $\mathscr{T}(G ; X)$.

LEMMA 3.1. Let $\left(\prod_{b \in B} \mathscr{T}\left(\pi^{-1}(b) ; X\right), \Gamma\right)$ be the continuous field of $C^{*}$-algebras from $\S 1$, and identify $\mathscr{T}(G ; X)$ with the $C^{*}$-algebra of sections of $\left(\prod_{b \in B} \mathscr{T}\left(\pi^{-1}(b) ; X\right), \Gamma\right)$. Then under this identification, the commutator ideal $\mathscr{C}(G ; X)$ of $\mathscr{T}(G ; X)$ corresponds to the ideal of sections that map $b$ into $\mathscr{C}\left(\pi^{-1}(b) ; X\right)$ for every $b \in B$.

Proof. This follows immediately from [3, Lemma 10.4.2].

Proposition 3.2. There exists a short exact sequence

$$
0 \longrightarrow \mathscr{C}(G ; X) \longrightarrow \mathscr{T}(G ; X) \stackrel{\sigma}{\longrightarrow} C(G) \longrightarrow 0,
$$

where $\sigma$ has the property that $\sigma\left(P M_{\phi}\right)=\phi$ for all $\phi$ in $C(G)$.

Proof. Define $\xi: C(G) \longrightarrow \mathscr{T}(G ; X) / \mathscr{C}(G ; X)$ by $\xi(\phi)=P M_{\phi}+$ $\mathscr{C}(G ; X)$. For all $\phi, \psi$ in $C(G), \xi(\phi \psi)-\xi(\phi) \xi(\psi)=\left(P M_{\phi \psi}-\right.$ $\left.P M_{\phi} P M_{\psi}\right)+\mathscr{C}(G ; X)$. Since $P^{b} M_{\phi^{b} \psi^{b}}-P^{b} M_{\phi^{b}} P^{b} M_{\psi^{b}} \in$ $\mathscr{C}\left(\pi^{-1}(b) ; X\right)$ for each $b \in B([7]), P M_{\phi \psi}-P M_{\phi} P M_{\psi} \in \mathscr{C}(G ; X)$ by Lemma 3.1, and thus $\xi$ is an homomorphism. Clearly $\xi$ is onto; I claim that $\xi$ is actually an isomorphism.

Choose $\phi \in \operatorname{ker} \xi$. Then $P M_{\phi} \in \mathscr{C}(G ; X)$ and $P^{b} M_{\phi^{b}}$ is in $\mathscr{C}\left(\pi^{-1}(b) ; X\right)$ for every $b$ in $B$. The results of [7] imply that $\phi^{b}$ is identically zero for all $b$, whence $\phi$ itself is zero. Our choice of $\phi$ in $\operatorname{ker} \xi$ was arbitrary, so $\operatorname{ker} \xi=0$ and $\xi$ is an isomorphism. If we define $\sigma$ to be the composition of $\xi^{-1}$ and the quotient map from $\mathscr{T}(G ; X)$ to $\mathscr{T}(G ; X) / \mathscr{C}(G ; X)$, the result follows. 
We apply the six-term exact sequence in $K$-theory to the short exact sequence in Proposition 3.2 to obtain

$$
\begin{array}{ccc}
K_{0}(\mathscr{C}(G ; X)) & \longrightarrow K_{0}(\mathscr{T}(G ; X)) & K_{0}(C(G)) \\
\partial_{1}^{a} \uparrow & \downarrow \partial_{0}^{a} \\
K_{1}(C(G)) & \longleftarrow K_{1}(\mathscr{T}(G ; X)) \longleftarrow K_{1}(\mathscr{C}(G ; X)) .
\end{array}
$$

Definition. The analytical index of $\mathscr{T}(G ; X)$ is the $\mathbb{Z}_{2}$-graded homomorphism

$$
\partial_{*}^{a}: K_{*}(C(G)) \longrightarrow K_{1-*}(\mathscr{C}(G ; X)) .
$$

4. The topological index. In this section we define a topological index for the Toeplitz algebra $\mathscr{T}(G ; X)$. We adopt [1] and [7] as references for the facts about $K$-theory that we need.

The continuous map $\pi: G \longrightarrow B$ gives rise to a six-term exact sequence

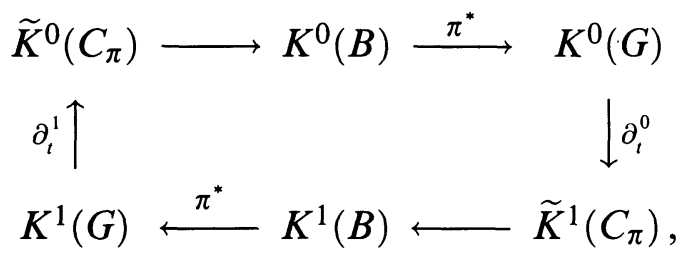

where $\widetilde{K}^{*}\left(C_{\pi}\right)$ denotes the reduced $K$-theory of the mapping cone $C_{\pi}$ of $\pi$.

Definition. The topological index is the $\mathbb{Z}_{2}$-graded homomorphism

$$
\partial_{t}^{*}: K^{*}(G) \longrightarrow \widetilde{K}^{1-*}\left(C_{\pi}\right)
$$

We end this section by reinterpreting the topological index so that in the next section we can identify the topological index and the analytical index.

Let $Z_{\pi}$ denote the mapping cylinder of $\pi$. The inclusion map from $B$ to $Z_{\pi}$ is a homotopy equivalence, so $K^{*}\left(Z_{\pi}\right)$ is isomorphic to $K^{*}(B)$. Also, $\widetilde{K}^{*}\left(C_{\pi}\right) \cong K^{*}\left(Z_{\pi} \backslash G\right)$. Finally, using the natural isomorphism between the $K$-theory of topological spaces and the $K$ theory of commutative $C^{*}$-algebras, the six-term exact sequence that defines the topological index can be identified with the following sequence: 


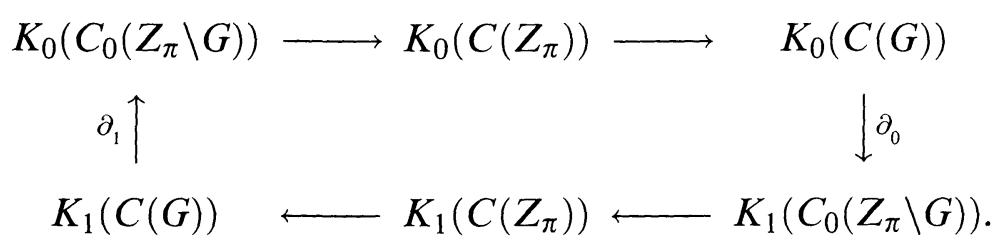

5. An index theorem. In this section, we identify the analytical index and the topological index. We also compute the $K$-theory of $\mathscr{C}(G ; X)$.

For any $C^{*}$-algebra $\mathscr{B}$, let $I \mathscr{B}$ denote the $C^{*}$-algebra of continuous functions from the unit interval into $\mathscr{B}$. Define

$$
\mathscr{A}(G ; X)=\{(\phi, F) \in C(B) \oplus I \mathscr{T}(G ; X): \alpha(\phi)=F(0)\} .
$$

Note that $\mathscr{A}(G ; X)$ is a pullback of $C(B)$ and $I \mathscr{T}(G ; X)$; this algebra provides the link between the analytical index and the topological index.

It follows immediately from the definition of the mapping cylinder $Z_{\pi}$ that $C\left(Z_{\pi}\right)$ is a pullback of $C(B)$ and $I C(G)$; specifically,

$$
C\left(Z_{\pi}\right) \cong\left\{(\phi, f) \in C(B) \oplus I C(G): \pi^{*}(\phi)=f(0)\right\} .
$$

Via this isomorphism, we define a homomorphism $\kappa: \mathscr{A}(G ; X) \longrightarrow$ $C\left(Z_{\pi}\right)$ by $\kappa(\phi, F)=(\phi, \hat{\sigma}(F))$, where $\hat{\sigma}$ is defined by the formula $\hat{\sigma}(F)(t)=\sigma(F(t))$.

Proposition 5.1. The homomorphism $\kappa$ induces an isomorphism $\kappa_{*}: K_{*}(\mathscr{A}(G ; X)) \longrightarrow K_{*}\left(C\left(Z_{\pi}\right)\right)$.

Proof. Let $\pi_{1}$ be the natural projection map from $\mathscr{A}(G ; X)$ to $C(B)$. Then

$$
\begin{aligned}
\operatorname{ker} \pi_{1} & =\{(\phi, F) \in C(B) \oplus I \mathscr{T}(G ; X): F(0)=\phi=0\} \\
& \cong C_{0}((0,1], \mathscr{T}(G ; X)) .
\end{aligned}
$$

Thus $K_{*}\left(\operatorname{ker} \pi_{1}\right)$ is identically zero, whence $\left(\pi_{1}\right)_{*}: K_{*}(\mathscr{A}(G ; X)) \longrightarrow$ $K_{*}(C(B))$ is an isomorphism. Next, the inclusion of $B$ into $Z_{\pi}$ is a homotopy equivalence, so the restriction map $r: C\left(Z_{\pi}\right) \longrightarrow C(B)$ induces an isomorphism in $K$-theory. Since $\pi_{1}$ is the composition of $\kappa$ and $r$, and since $\pi_{1}$ and $r$ induce isomorphisms in $K$-theory, so does $\kappa$.

Proposition 5.2. Define $\tau: \mathscr{A}(G ; X) \longrightarrow \mathscr{T}(G ; X)$ by $\tau(\phi, F)=$ $F(1)$. Then $\tau$ induces an isomorphism in $K$-theory. 
Proof. We proved in Proposition 5.1 that $\pi_{1}$ induces an isomorphism in $K$-theory. We know from $\S 2$ that $\alpha$ also induces an isomorphism in $K$-theory. Thus the composition $\alpha \pi_{1}$ gives rise to an isomorphism from $K_{*}(\mathscr{A}(G ; X))$ to $K_{*}(\mathscr{T}(G ; X))$. Now, $\left(\alpha \pi_{1}\right)(\phi, F)=$ $F(0) \neq \tau(\phi, F)$. However, $\alpha \pi_{1}$ is homotopic to $\tau$, so $\tau_{*}$ is also an isomorphism.

Define $\sigma_{1}: \mathscr{A}(G ; X) \longrightarrow C(G)$ by $\sigma_{1}(\phi, F)=\sigma(F(1))$. We then have the following commutative diagram with exact rows:

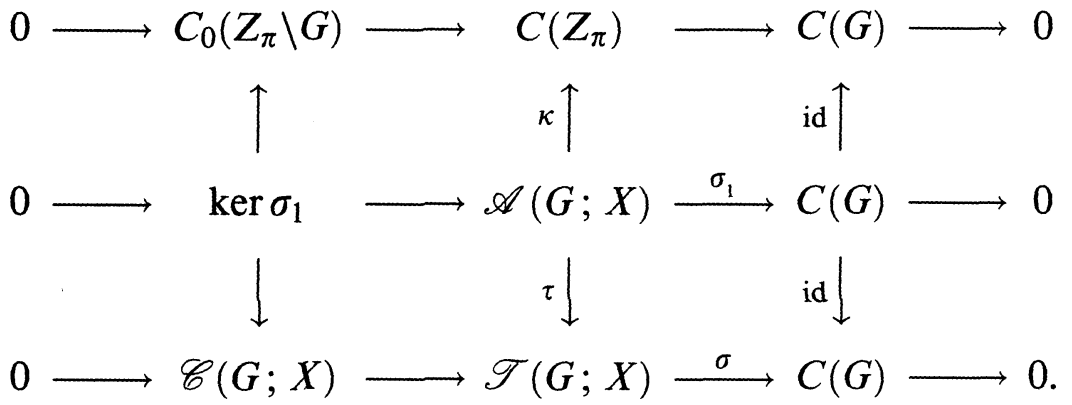

The homomorphisms $\kappa$ and $\tau$ induce isomorphisms in $K$-theory, so the six-term exact sequence in $K$-theory and the five lemma imply that the vertical maps on the left induce isomorphisms in $K$-theory. Thus we have an isomorphism from $K_{*}(\mathscr{C}(G ; X))$ to $K_{*}\left(C_{0}\left(Z_{\pi} \backslash G\right)\right) \cong$ $\widetilde{K}^{*}\left(C_{\pi}\right)$, and hence we have computed the $K$-theory of $\mathscr{C}(G ; X)$ :

THEOREM 5.3. $K_{*}(\mathscr{C}(G ; X)) \cong \widetilde{K}^{*}\left(C_{\pi}\right)$.

The naturality of the connecting maps in $K$-theory allows us to use the diagram above to identify the analytical and topological indices.

THEOREM 5.4. The following diagram commutes:

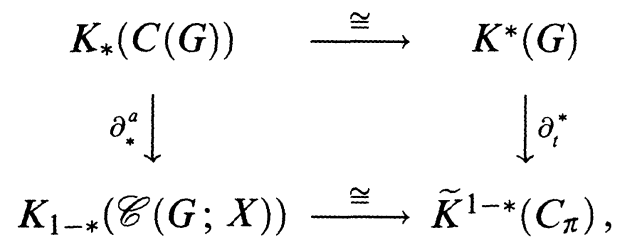

where the lower horizontal map is the isomorphism in Theorem 5.3 and the upper horizontal map is the natural isomorphism.

6. The smoothed Toeplitz algebra. In this section, we consider a "smoothed" version $\mathscr{T}_{\varepsilon}(G ; X)$ of the Toeplitz algebra $\mathscr{T}(G ; X)$. There are several reasons for doing this; we refer the reader to [4] for more information. 
Definition. Let $\varepsilon>0$. The smoothed Toeplitz algebra, denoted $\mathscr{T}_{\varepsilon}(G ; X)$, is the $C^{*}$-subalgebra of $\mathscr{L}\left(L^{2}(G)\right)$ generated by $\left\{P_{\varepsilon} M_{\phi}\right.$ : $\phi \in C(G)\}$, where $P_{\varepsilon}=f_{\varepsilon}\left(\frac{1}{i} L_{X}\right)$ and $f_{\varepsilon}: \mathbb{R} \longrightarrow \mathbb{R}$ is defined by $f_{\varepsilon}(t)=\frac{1}{2}\left[1+t\left(\varepsilon+t^{2}\right)^{-1 / 2}\right]$.

We remark that the actual choice of $\varepsilon>0$ and function $f_{\varepsilon}$ is not crucial for most purposes; see [4] for a discussion of this matter.

We begin by assuming that $\exp (t X)$ is a closed subgroup of $G$. In this case the index theory of the smoothed Toeplitz algebra $\mathscr{T}_{\varepsilon}(G ; X)$ will be no different from that of $\mathscr{T}(G ; X)$. This is because the spectrum of $\frac{1}{i} L_{X}$ is discrete when $\exp (t X)$ is closed and therefore has a gap about zero.

When $\exp (t X)$ is not closed, the situation is quite different. The algebra $\mathscr{T}_{\varepsilon}\left(\mathbb{T}^{n} ; X\right)$ was studied in [5] for the case $n>1$ and $\exp (t X)$ a dense subgroup of $\mathbb{T}^{n}$. The authors of [5] proved that there is a short exact sequence

$$
0 \longrightarrow \mathscr{C}_{\varepsilon}\left(\mathbb{T}^{n} ; X\right) \longrightarrow \mathscr{T}_{\varepsilon}\left(\mathbb{T}^{n} ; X\right) \longrightarrow C\left(\mathbb{T}^{n}\right) \longrightarrow 0,
$$

where $\mathscr{C}_{\varepsilon}\left(\mathbb{T}^{n} ; X\right)$ denotes the commutator ideal of $\mathscr{T}_{\varepsilon}\left(\mathbb{T}^{n} ; X\right)$. They also proved that the connecting maps in the associated six-term $K$ theory exact sequence are isomorphisms. Thus $K_{*}\left(\mathscr{T}_{\varepsilon}\left(\mathbb{T}^{n} ; X\right)\right)=0$.

More generally, let $G$ be an arbitrary compact Lie group, and suppose that $\exp (t X)$ is not closed. The results of $\S 1$ apply, mutatis mutandis, to $\mathscr{T}_{\varepsilon}(G ; X)$. In particular, Corollary 1.4 is true. We use the Künneth formula and the Mayer-Vietoris sequence to conclude

Theorem 6.1. Let $G$ be a compact Lie group, let $X$ be a nonzero left-invariant vector field on $G$, and suppose that $\exp (t X)$ is not closed. Then $K_{*}\left(\mathscr{T}_{\varepsilon}(G ; X)\right)=0$.

Furthermore, the proof of Proposition 3.2 adapts to give a short exact sequence

$$
0 \longrightarrow \mathscr{C}_{\varepsilon}(G ; X) \longrightarrow \mathscr{T}_{\varepsilon}(G ; X) \longrightarrow C(G) \longrightarrow 0,
$$

so we have

COROLlaRY 6.2. Under the hypotheses of Theorem 6.1,

$$
K_{*}\left(\mathscr{C}_{\varepsilon}(G ; X)\right) \cong K^{1-*}(G) \text {. }
$$

Proof. Apply the six-term exact sequence in $K$-theory to the short exact sequence above to get $K_{*}\left(\mathscr{C}_{\varepsilon}(G ; X)\right) \cong K_{1-*}(C(G))$, and then use the isomorphism $K_{*}(C(G)) \cong K^{*}(G)$. 
We conclude by remarking that one can say more about the commutator ideal $\mathscr{C}_{\varepsilon}(G ; X)$. It is shown in [5] and [6] that $\mathscr{C}_{\varepsilon}\left(\mathbb{T}^{n} ; X\right)$ is isomorphic to the crossed-product $C^{*}$-algebra $C\left(\mathbb{T}^{n}\right) \rtimes \mathbb{R}$, where the flow that $X$ defines on $\mathbb{T}^{n}$ gives the action of $\mathbb{R}$. A straightforward argument using continuous fields shows that $\mathscr{C}_{\varepsilon}(G ; X)$ is isomorphic to a crossed product $C(G) \rtimes \mathbb{R}$.

\section{REFERENCES}

[1] B. Blackadar, K-Theory for Operator Algebras, Math. Sci. Res. Inst. Publ. No. 5, Springer, Berlin Heidelberg New York, 1986.

[2] R. Curto, P. Muhly and J. Xia, Toeplitz operators on flows, (to appear).

[3] J. Dixmier, $C^{*}$-Algebras, North-Holland, Amsterdam, 1977.

[4] R. Douglas, S. Hurder and J. Kaminker, The longitudinal cocycle and the index of Toeplitz operators, (to appear).

[5] R. Ji and J. Kaminker, The K-theory of Toeplitz extensions, J. Operator Theory, 19 (1988), 347-354.

[6] R. Ji and J. Xia, On the classification of commutator ideals, J. Funct. Anal., 78 (1988), 208-232.

[7] M. Karoubi, K-Theory, Springer, Berlin Heidelberg New York, 1978.

[8] P. Muhly, I. Putnam and J. Xia, On the K-theory of some $C^{*}$-algebras of Toeplitz and singular integral operators, (to appear).

[9] C. Schochet, Topological methods for $C^{*}$-algebras II: geometric resolution and the Künneth formula, Pacific J. Math., 98 (1987), 443-458.

[10] __ Topological methods for $C^{*}$-algebras III: axiomatic homology, Pacific J. Math., 114 (1984), 339-445.

[11] M. Takesaki, Theory of Operator Algebras I, Springer, Berlin Heidelberg New York, 1979.

Received October 11, 1990 and in revised form April 13, 1992.

TeXas Christian University

FORT WORTH, TX 76129 



\title{
PACIFIC JOURNAL OF MATHEMATICS
}

Founded by

\author{
E. F. BeCKeNBACH (1906-1982) F. Wolf (1904-1989)
}

\section{EDITORS}

\section{S. VARADARAJAN (Managing Editor) University of California Los Angeles, CA 90024-1555 vsv@math.ucla.edu \\ F. Michael Christ University of California Los Angeles, CA 90024-1555 christ@math.ucla.edu}

\section{Herbert Clemens}

University of Utah

Salt Lake City, UT 84112

clemens@math.utah.edu

\author{
THOMAS ENRIGHT \\ University of California, San Diego \\ La Jolla, CA 92093 \\ tenright@ucsd.edu \\ Nicholas ERcolani \\ University of Arizona \\ Tucson, AZ 85721 \\ ercolani@math.arizona.edu \\ R. FINN \\ Stanford University \\ Stanford, CA 94305 \\ finn@gauss.stanford.edu \\ VAUGHAN F. R. JONES \\ University of California \\ Berkeley, CA 94720 \\ vfr@math.berkeley.edu
}

\section{SUPPORTING INSTITUTIONS}

UNIVERSITY OF ARIZONA

UNIVERSITY OF BRITISH COLUMBIA

UNIVERSITY OF OREGON

CALIFORNIA INSTITUTE OF TECHNOLOGY

UNIVERSITY OF CALIFORNIA

UNIVERSITY OF MONTANA

UNIVERSITY OF NEVADA, RENO

NEW MEXICO STATE UNIVERSITY

OREGON STATE UNIVERSITY
SteVen KerckhofF

Stanford University

Stanford, CA 94305

spk@gauss.stanford.edu

MARTIN SCHARLEMANN

University of California

Santa Barbara, CA 93106

mgscharl@henri.ucsb.edu

HAROLD STARK

University of California, San Diego

La Jolla, CA 92093

\author{
STANFORD UNIVERSITY \\ UNIVERSITY OF HAWAII \\ UNIVERSITY OF UTAH \\ WASHINGTON STATE UNIVERSITY \\ UNIVERSITY OF WASHINGTON
}

UIV 


\section{PACIFIC JOURNAL OF MATHEMATICS}

Volume $158 \quad$ No. $1 \quad$ March 1993

Determinant identities

GEORGE W. Eyre ANDrEWS and WiLliam H. Burge

A spectral theory for solvable Lie algebras of operators

E. BOASSO and ANGEL RAFAEL LAROTONDA

Simple group actions on hyperbolic Riemann surfaces of least area

S. Allen Broughton

Duality for finite bipartite graphs (with an application to $\mathrm{II}_{1}$ factors)

MARIE CHODA

Szegő maps and highest weight representations

MARK GREGORY DAVIDSON and Ron STANKE

Optimal approximation class for multivariate Bernstein operators

ZEEV DitZIAN and XINLONG ZHOU

Witt rings under odd degree extensions

ROBERT FITZGERALD

Congruence properties of functions related to the partition function

ANTHONY D. FORBES

Bilinear operators on $L^{\infty}(G)$ of locally compact groups

Colin C. GRAham and Anthony To-Ming LAU

Nonuniqueness of the metric in Lorentzian manifolds

GEOFFREY K. MARTIN and GERARD THOMPSON

Index theory and Toeplitz algebras on one-parameter subgroups of Lie 189 groups

EFTON PARK 